used in $13.2 \%$ of palsied patients was not significantly different from the $10.2 \%$ in the normal population. The prevalence of maternal primiparity $(39.6 \%)$ among mothers of affected babies was no greater than that expected from national data. Big babies weighing $>3500 \mathrm{~g}(18.9 \%)$ were fewer in number in the study group than in the general population $(38.6 \%)$. This study shows that the risk of birth trauma is no higher in children with permanent 'congenital' facial palsy than in the general population. An intrauterine rather than a traumatic birth etiology is suggested. (Laing JHE et al. Is permanent congenital facial palsy caused by birth trauma? Arch Dis Child January 1996;74:56-58). (Respond: Mr Hamish Laing, 13 Princess Road, Primrose Hill, London NW1 8JR. Reprints: The Secretary, RAFT Institute of Plastic Surgery, Mount Vernon Hospital, Northwood, Middlesex HA6 2RN, UK).

COMMENT. In this large retrospective study, infants born with facial palsy were no more likely to have required forceps to assist delivery, and other risk factors for traumatic birth were not more prevalent than expected from national data. A commonly held assumption that birth trauma is frequently responsible for permanent congenital facial palsy appears to be false.

The authors of a study of 5 cases of unilateral congenital facial palsy identified in a retrospective review at the Massachusetts Eye and Ear Infirmary, Boston, MA, point out that spontaneous recovery is expected within 4 weeks in $90 \%$ of traumatic cases, and the prevalence of atraumatic developmental etiologies has been underemphasized in persistent congenital unilateral facial palsies. (Shapiro NL et al. Congenital unilateral facial paralysis. Pediatrics February 1996;97:261-265). The differential diagnosis of developmental cases includes: Mobius syndrome, cardiofacial syndrome, Goldenhar's syndrome (hemifacial microsomia), Poland's syndrome, DiGeorge syndrome, Albers-Schonberg disease, sclerostosis, trisomy 13, trisomy 18, and thalidomide embryopathy. In addition to these complex cases, isolated facial palsies may be complete or affect only the upper or lower lip. Electrophysiological and CT studies and especially electroneuronography (ENOG) are essential in the early differentiation of traumatic facial paralyses and those of developmental origin.

\title{
ETIOLOGY OF SENSORINEURAL DEAFNESS
}

Children with a bilateral sensorineural hearing impairment averaging $30 \mathrm{dBHL}$ or or more were investigated for possible congenital or intrauterine infection as causes at the Centre for Audiology, Manchester University, UK. Of a total of 339 cases studied, $23 \%$ had a positive family history of deafness in parents or siblings (genetic group); $13 \%$ had suffered from birth asphyxia, respiratory distress syndrome, intraventricular hemorrhage, infections, or hyperbilirubinemia (perinatal group); $8 \%$ had congenital rubella or cytomegalovirus infection; $7 \%$ had a history of bacterial meningitis; $5 \%$ chromosomal anomalies; 5\% were diagnosed with Waardenburg, Hurler, Hunter, Klippel Feil, or other syndromes; and 34\% were of unknown cause. Autosomal recessive inheritance was probably responsible for most cases of unknown etiology. (Das VK. Aetiology of bilateral sensorineural hearing impairment in children: a 10 year study. Arch Dis Child January 1996;74:8-12). (Respond: Dr VK Das, Centre for Audiology, Education of the Deaf and Speech Pathology, The University, Oxford Road, Manchester M13 9PL, UK).

COMMENT. The authors are critical of the present screening 
arrangements for hearing impaired children. They advocate early identification by screening, not only in the at risk children but universally. Testing only 'at risk' children leads to identification of less than half those affected. Regular school screening should help in diagnosis, and genetic counselling might lead to a reduction of numbers in the largest etiological group of cases. Hearing tests are important in the neurologic evaluation of children presenting with learning or attention deficit disorders, especially in those with a positive family history of deafness or a prior risk factor illness.

\section{MUSCLE DISORDERS}

\section{ADHALIN DEFICIENCY IN MUSCULAR DYSTROPHY}

A 13-year-old boy previously diagnosed with Becker's muscular dystrophy and dilated cardiomyopathy was studied at the University of Wisconsin, Madison, and the University of Iowa College of Medicine, Iowa City, and was found to have a deficiency of the dystrophin-associated glycoprotein, adhalin. He was asymptomatic until 9 years of age, when proximal weakness developed. He had flexion contractures at the ankles, hypertrophy of calf muscles, and Gower's sign. The serum creatine kinase level was 11,560 U/L. Both his sister and mother had normal CK. There was no consanguinity. Analysis of dystrophin from the biceps by Western blot was normal. Congestive heart failure required heart transplantation. Immunostaining in both skeletal and cardiac muscle showed normal dystrophin, whereas adhalin was reduced in skeletal muscle and absent in cardiac muscle. (Fadic R, Lotz BP et al. Brief report: Deficiency of a dystrophin-associated glycoprotein (adhalin) in a patient with muscular dystrophy and cardiomyopathy. N Engl I Med Feb 8, 1996;334:362-365). (Reprints: Dr Lotz, Department of Neurology, University of Wisconsin Hospital and Clinics, 600 Highland Ave, Madison, WI 53792).

COMMENT. Adhalin deficiency is an autosomal recessive disorder and is indistinguishable from the dystrinopathies by clinical presentation and muscle pathology. The authors propose that constituents of the dystrophinglycoprotein complex (adhalin) and merosin should be analysed histochemically in all patients with histological findings suggestive of a dystrophinopathy and with normal muscle dystrophin. The dystrophinassociated glycoprotein was named "adhalin" from the Arabic adhal (muscle). It has been linked in North African populations to a gene in chromosome 13q, but the deficiency is genetically heterogeneous. The adhalin gene has been mapped to chromosome 17q. See Ped Neur Briefs Oct 1995, pp73-74, for reference to a further case report of primary adhalin deficiency in a 16-yearold African-American girl with childhood-onset limb-girdle muscular dystrophy.

\section{FEBRILE CONVULSIONS}

\section{FEBRILE SEIZURE DURATION AND TEMPORAL LOBE EPILEPSY}

Clinical features of febrile seizures and EEG findings were compared in patients who did and did not develop later afebrile seizures among six selected families and 59 family members with febrile convulsions examined at the Department of Clinical Neurological Sciences, University of Western Ontario, London, Ontario. All six probands developed epilepsy, 5 with temporal lobe epilepsy (TLE), after onset of febrile convulsions (FC). Of 59 family members 